\title{
MILCA, a modified internal linear combination algorithm to extract astrophysical emissions from multifrequency sky maps
}

\author{
G. Hurier ${ }^{1,2}$, J. F. Macías-Pérez ${ }^{1}$, and S. Hildebrandt ${ }^{1,3}$ \\ ${ }^{1}$ Laboratoire de Physique Subatomique et de Cosmologie, Université Joseph Fourier Grenoble I, CNRS/IN2P3, \\ Institut National Polytechnique de Grenoble, 53 rue des Martyrs, 38026 Grenoble Cedex, France \\ e-mail: ghurier@ias.u-psud.fr \\ 2 Institut d'Astrophysique Spatiale, CNRS (UMR8617), Université Paris-Sud 11, Bâtiment 121, 91405 Orsay, France \\ 3 Jet Propulsion Laboratory, California Institute of Technology, 4800 Oak Grove Drive, Pasadena, California, USA
}

Received 14 May 2013 / Accepted 23 July 2013

\begin{abstract}
This analysis of current cosmic microwave background (CMB) experiments is based on the interpretation of multifrequency sky maps in terms of different astrophysical components and it requires specifically tailored, component separation algorithms. In this context, internal linear combination (ILC) methods have been extensively used to extract the CMB emission from the WMAP multifrequency data. We present here a modified internal linear component algorithm (MILCA) that generalizes the ILC approach to the case of multiple astrophysical components for which the electromagnetic spectrum is known. In addition, MILCA corrects for the intrinsic noise bias in the standard ILC approach and extends it to a hybrid space-frequency representation of the data. It also allows us to use external templates to minimize the contribution of extra components but still using only a linear combination of the input data. We applied MILCA to simulations of the Planck satellite data at the frequency bands from $100 \mathrm{GHz}$ to $857 \mathrm{GHz}$. We explore the possibility of reconstructing the Galactic molecular CO emission and the thermal Sunyaev-Zeldovich effect from the Planck maps. We conclude that MILCA is able to accurately estimate those emissions, and it has been successfully used for this purpose within the Planck collaboration.
\end{abstract}

Key words. methods: data analysis - techniques: image processing - cosmic background radiation

\section{Introduction}

Multifrequency comic microwave background (CMB) experiments, such as the WMAP (Bennett et al. 2003) and Planck (Planck Collaboration 2006) missions, observes a mixture of astrophysical emissions. Most of them follow different emission laws (e.g., CMB anisotropies, thermal Sunyaev-Zeldovich (tSZ) effect, Sunyaev \& Zeldovich 1972, thermal dust, cosmic infrared background (CIB), synchrotron, molecular cloud emission, etc.). It is thus possible to separate the mixed astrophysical sources by taking advantage of the specific emission laws of each astrophysical component. The separation of astrophysical sources from a set of multichannel observations of the millimeter and submillimeter sky is an important step in the scientific exploitation of such data. In this context, several methods have been developed.

These component separation techniques can be separated into various categories depending on the degree and nature of the prior information used. For example, maximum likelihood techniques (e.g., Commander, Eriksen et al. 2008) and maximum of entropy methods (see e.g. Hobson et al. 1998; Stolyarov et al. 2002) assume that the electromagnetic spectrum of the different components is known or that it can be easily parametrized. By contrast, blind (or semi-blind) component separation methods such as, spectral matching ICA (Cardoso et al. 2008; Delabrouille et al. 2003), PolEMICA (Aumont \& Macías-Pérez 2007), FastICA (Maino et al. 2002), CCA (Bonaldi et al. 2006), or GMCA (Bobin et al. 2008) exploit the spectral and spatial diversity of the components and need no a priori. In a different way, ILC (internal linear combination) techniques (Bennett et al. 2003; Eriksen et al. 2004; Remazeilles et al. 2011) aim to preserve an astrophysical component for which the electromagnetic spectrum is known, by minimizing the variance in the reconstructed signal.

Standard ILC methods are very popular because of their simplicity and robustness. However, as discussed by Vio \& Andreani (2008), they are biased by the noise contribution to the variance of the final map. This bias reduces the efficiency of the algorithm in poor signal-to-noise conditions. Furthermore, they do not fully exploit the spatial and spectral diversity to minimize the contribution from other sky components for which the electromagnetic spectrum is poorly, or not at all, known.

The ILC methods also assume that the component of interest is statistically independent of the other ones. If the statistical independence assumption can be made for the CMB, it is not the case for all the astrophysical components of the millimeter and submillimeter sky. Indeed, galactic foregrounds, such as thermal dust, synchrotron, and molecular cloud emission, are highly correlated over the sky. Such correlations can also be observed for extragalactic components like tSZ and extragalactic point sources.

We present, in this article, the modified internal linear combination algorithm (MILCA) that generalizes standard ILC techniques to multiple constraints in order to reduce the level of contamination by other astrophysical emissions. We show that MILCA also corrects for the noise bias of the standard 
ILC solution and blindly minimizes the contribution from unknown or poorly constrained sky components.

The paper is organized as follows. In Sect. 2, we present our modeling the millimeter and submilimeter sky. Then, in Sect. 3, we address the problem of generalizing the ILC algorithm to multiple constraints. Section 4 discusses possible bias in the ILC algorithm. In Sect. 5, we propose modifications to the standard ILC estimator to minimize the instrumental noise and contamination from other astrophysical components. Finally, we apply MILCA to Planck simulated datasets, focusing on the extraction of the tSZ effect(Sect. 6) and molecular cloud emission (Sect. 7).

It is important to note that MILCA has already been used by the Planck collaboration to produce tSZ $y$-maps for validating the catalog of candidates (Planck Collaboration 2011d,i), for the extraction of the cluster pressure profiles (Planck Collaboration 2013b), for other physical analyses (Planck Collaboration 2013f,c) and for comparison with other experiments (Planck Collaboration 2013a). MILCA has also been used to produce tSZ maps in Planck Collaboration (2011d,i, 2013f,a,e,m,l).

MILCA has also been applied to the Planck bolometer maps at 100,217 , and $353 \mathrm{GHz}$ in order to extract maps of the emission of the first three CO rotational transitions $J=1-0, J=2-1$, and $J=3-2$ (Planck Collaboration 2013h). These maps were released by the Planck collaboration and have been extensively compared with external data in Planck Collaboration (2013h).

\section{Simulation of the microwave sky seen by Planck}

\subsection{The Planck mission and the submillimeter sky}

The Planck mission (Planck Collaboration 2011a, 2013g) is the third space mission, after the COBE (Smoot et al. 1992; Bennett et al. 1996) and the WMAP (Hinshaw et al. 2003, 2009; Bennett et al. 2003) missions, to be devoted to the study of the microwave and submm emissions, and especially to CMB. Planck, as other $\mathrm{CMB}$ experiments, observes the sky emission in different frequency bands. In particular, Planck has two instruments: the Low Frequency Instrument (LFI, Bersanelli et al. 2010; Mennella et al. 2011), which has three frequency bands centered at 30, 44, and $70 \mathrm{GHz}$, and the High Frequency Instrument (HFI, Lamarre et al. 2010; Planck Collaboration 2011b), which has six frequency bands centered at 100, 143, 217, 353, 545, and $857 \mathrm{GHz}$. The large number of detectors and their high sensitivity, make the Planck mission the first CMB experiment expected to be limited by our knowledge of the foreground emissions rather than by instrumental noise.

At the Planck frequencies, the main astrophysical emissions are the diffuse Galactic free-free, synchrotron, and thermal dust (Planck Collaboration 2006, 2011h) emissions; the anomalous microwave emission (AME, Planck Collaboration $2011 \mathrm{~g}$ ); the molecular Galactic emissions (mainly ${ }^{12} \mathrm{CO}$ in the 100, 217, and $353 \mathrm{GHz}$ bands, Planck Collaboration 2013h); the emission from Galactic and extragalactic point sources (radio and infrared sources, Planck Collaboration 2013d, 2011c); the CIB (Planck Collaboration 2011f); the zodiacal light emission (Planck Collaboration 2013i); and the thermal SunyaevZeldovich effect (Sunyaev \& Zeldovich 1972) in clusters of galaxies (Planck Collaboration 2011d, 2013m).

\subsection{Simulations of the submillimeter sky}

We have simulated the microwave and submillimeter sky observed by Planck using a set of template maps to reproduce astrophysical components. Within those components, we include $\mathrm{CMB}$, diffuse Galactic emissions (synchrotron, free-free and thermal dust), Galactic CO emission, Galactic and extragalactic point sources, CIB and $\mathrm{tSZ}$ emissions. We did not include in the simulation the Anomalous Microwave Emission (AME) for which a template is not available and that we expect to be subdominant for the following analyses based on data from 100 to $857 \mathrm{GHz}$.

The CMB was simulated using a random correlated gaussian field as defined by the Planck best-fit CMB angular power spectrum (Planck Collaboration 2013j,k). The thermal dust emission was modeled using the reprocessed IRAS maps at $100 \mu \mathrm{m}$ (IRIS, Miville-Deschênes \& Lagache 2005) extrapolated to the Planck frequencies assuming a grey body emission law (e.g. Desert et al. 1990) with constant temperature, $T_{\text {dust }}=18 \mathrm{~K}$ and spectral index $\beta_{\text {dust }}=1.8$.

The synchrotron emission was simulated using the $408 \mathrm{MHz}$ full-sky map from Haslam et al. (1981) corrected for free-free emission assuming an emission law with a spatially constant spectral index of -1.0 in terms of flux density (Davies et al. 1996). The Galactic free-free emission was modeled using the $\mathrm{H}_{\alpha}$ full-sky map (Finkbeiner 2003) and an electron temperature of $7000 \mathrm{~K}$ as proposed by Dickinson et al. (2003) and a spatially constant spectral index of -0.1 in terms of flux density. The CO rotational transition line emission was modeled using the $J=1-0$ CO survey from Dame et al. (2001). The CO transmission in Planck's sky maps have been simulated assuming $10 \%$ fluctuations on the bandpass amplitude (Planck Collaboration 2013h).

We also add radio point sources using the sources in the NVSS catalog (Condon et al. 1998) and extrapolating their fluxes to the Planck frequencies assuming a random spectral index for each source derived from a Gaussian distribution with a mean of -0.5 and a standard deviation of 0.2 in terms of flux density (Planck Collaboration 2011e).

Extragalactic infrared (IR) astrophysical emissions can be decomposed into two categories, a poissonian contribution, composed by point sources, and a diffuse contribution, the clustered CIB. To model IR point sources, we used a randomly distributed sources with random spectral index for each source (with a mean of 2.8 and a standard deviation of 0.3 in terms of spectral density as found in Planck Collaboration 2011e). For the CIB emission, we assumed a random correlated gaussian distribution following the CIB power spectra as measured by Planck (Planck Collaboration 2011f) and constant correlation factors across multipoles. We do not account for the nongaussianity of the CIB (see e.g. Lacasa et al. 2012) in our model.

The tSZ emission is produced by the hot and dense gas of electrons, such gas can be found in galaxy clusters or in the WHIM. In this study we only account for the emission from X-ray known galaxy clusters. To do so, the tSZ was simulated using a universal electron pressure profile from Arnaud et al. (2010) and physical parameters for clusters from the MCXC catalog (Piffaretti et al. 2011).

The simulations of the Planck frequency channels (from 100 to $857 \mathrm{GHz}$ ) we produced are presented in Fig. 1. It is important to note that the simulations presented in this paper are simplified as they do not account for spatial variations in the SED of the Galactic synchrotron and thermal dust emissions. However, these simplified simulations allow us to present the main characteristics of the MILCA procedure. A discussion of the application of MILCA to more realistic sky emission simulations constructed from the Planck sky model (PSM, Delabrouille et al. 2013), can be found in Planck Collaboration (20131). 

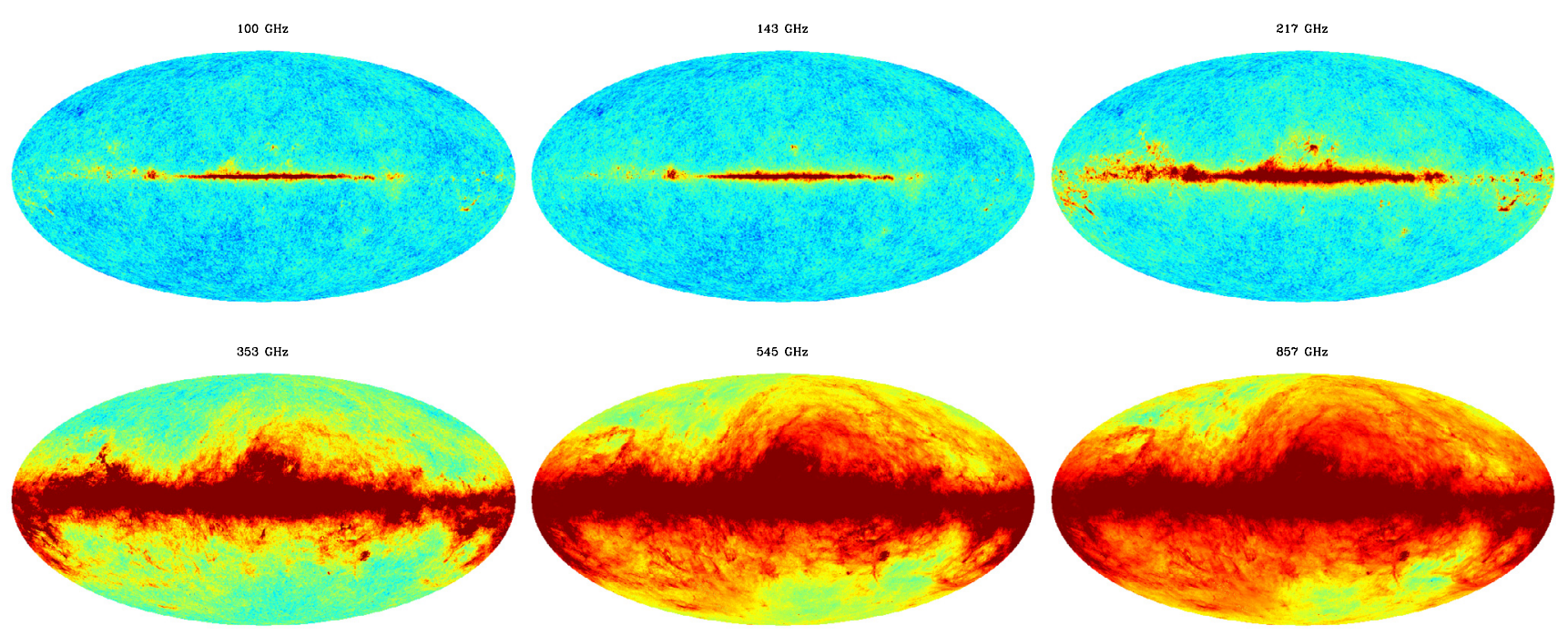

Fig. 1. Simulation of the submillimeter sky for, from left to right and top to bottom; 100, 143, 217, 353, 545, and $857 \mathrm{GHz}$ Planck channels. See Sect. 2 for more details.

For each Planck channel, maps of the instrumental noise have been produced and added to the sky emission simulated maps, with the expected levels for the Planck mission (Planck Collaboration 2013g). The noise is assumed to be Gaussian and white but anisotropic as a consequence of the Planck scanning strategy (Planck Collaboration 2006).

\section{Generalizing ILC method}

The total emission observed at a given frequency channel map, $T_{i}$, can be expressed as the superposition of astrophysical components and instrumental noise

$T_{i}(p)=\sum_{j=1}^{N_{\mathrm{s}}} \mathcal{A}_{i j}(p) S_{j}(p)+n_{j}(p)$,

where $p$ denotes the pixel number. $S_{j}$ are the maps of the $N_{\mathrm{s}}$ astrophysical components (CMB and foregrounds) in the data and $n_{j}$ is the instrumental noise. $\mathcal{A}_{i j}$ is the mixing matrix given by

$\mathcal{A}_{i j}=\int F_{j}(v) H_{i}(v) \mathrm{d} v$,

with $F_{j}(v)$ the spectral dependence of the $j$ th component and $H_{i}(v)$ the spectral response of the $i$ th channel.

Assuming that the mixing matrix is constant across the sky or constant across a given set of pixels, Eq. (1) can be written in a more compact manner as

$\boldsymbol{T}(p)=\mathcal{A S}(p)+N(p)$,

where $\boldsymbol{T}$ and $\boldsymbol{N}$ are vectors containing the total and noise maps for the $N_{\text {obs }}$ observation channels, $\mathbf{S}$ is a matrix corresponding to the maps of the $N_{\mathrm{s}}$ astrophysical components. $\mathcal{A}$ is the mixing matrix with dimensions $N_{\text {obs }} \times N_{\mathrm{s}}$.

\subsection{Internal Linear Combination algorithm}

The Internal Linear Combination algorithm (Bennett et al. 2003) assumes that the astrophysical component to be reconstructed,
$S_{\mathrm{c}}$, can be obtained as a linear combination of the input observational maps

$\hat{S}_{\mathrm{c}}(p)=\boldsymbol{w}^{\boldsymbol{T}} \boldsymbol{T}=\sum_{i} w_{i} T_{i}(p)$

The weights, $\boldsymbol{w}^{\boldsymbol{T}}=\left(w_{1}, \ldots, w_{N_{\mathrm{obs}}}\right)$, of the linear combination are computed by minimizing the variance of the output map,

$$
\begin{aligned}
\operatorname{Var}\left(\hat{S}_{\mathrm{c}}\right) & =\frac{1}{N_{\text {pix }}} \sum_{p=1}^{N_{\text {pix }}} \hat{S}_{\mathrm{c}}^{2}(p)-\left(\sum_{p=1}^{N_{\text {pix }}} \hat{S}_{\mathrm{c}} / N_{\text {pix }}\right)^{2} \\
& =\boldsymbol{w}^{\boldsymbol{T}}\left\langle\boldsymbol{T} \boldsymbol{T}^{\boldsymbol{T}}\right\rangle \boldsymbol{w}=\boldsymbol{w}^{\boldsymbol{T}} \boldsymbol{C}_{\boldsymbol{T}} \boldsymbol{w}
\end{aligned}
$$

under constraints on the emission spectrum of $S_{\mathrm{c}}$. The matrix $C_{T}$ is the covariance matrix of the observed maps, $\boldsymbol{T}$, averaged across pixels.

For the CMB and assuming that the maps are given in thermodynamic temperature units, the constraint simply reads as (Bennett et al. 2003; Eriksen et al. 2004)

$g=\sum_{i} \boldsymbol{w}_{i}=1$

More generally, for an astrophysical component with emission spectrum, $\boldsymbol{f}_{\boldsymbol{c}}$, the constraints can be written as

$g=\boldsymbol{w}^{T} \boldsymbol{f}_{c}=1$.

We stress that $\boldsymbol{f}_{c}$ is related to the mixing matrix, $\mathcal{A}$, by $\boldsymbol{f}_{\boldsymbol{c}}=$ $\mathcal{A} \boldsymbol{e}_{\boldsymbol{c}}$, where $\boldsymbol{e}_{\boldsymbol{c}}$ is an $N_{\mathrm{s}}$ dimension vector for which all elements are set to zero except the $c$-th element that is set to one.

\section{Linear system solution}

Weights can be computed solving a linear system using Lagrange multipliers, $\lambda$,

$$
\begin{array}{r}
\boldsymbol{\nabla} V\left(S_{\mathrm{c}}\right)-\lambda \boldsymbol{\nabla} g=0 \\
\boldsymbol{g}=\boldsymbol{f}_{\boldsymbol{c}}^{\boldsymbol{T}} \boldsymbol{w}=1 .
\end{array}
$$


The final linear system can be expressed in the form

$$
\left(\begin{array}{cc}
2 . C_{T} & -f_{c} \\
f_{c}{ }^{T} & 0
\end{array}\right)\left(\begin{array}{l}
w \\
\lambda
\end{array}\right)=\left(\begin{array}{l}
0 \\
1
\end{array}\right) \text {. }
$$

Solving the linear system we obtain

$\widehat{w}=C_{T}^{-1} f_{c}\left(f_{c}{ }^{T} C_{T}^{-1} f_{c}\right)^{-1}$

and then

$\widehat{S}_{\mathrm{c}}(p)=\left(\boldsymbol{f}_{\boldsymbol{c}}{ }^{T} \boldsymbol{C}_{\boldsymbol{T}}^{-1} \boldsymbol{f}_{\boldsymbol{c}}\right)^{-1} \boldsymbol{f}_{\boldsymbol{c}}^{T} \boldsymbol{C}_{\boldsymbol{T}}^{-1} \boldsymbol{T}(p)$.

In the case of the CMB emission Eq. (11) reduces to

$\widehat{S}_{\mathrm{c}}(p)=\frac{1^{T} \boldsymbol{C}_{\boldsymbol{T}}^{-1} \boldsymbol{T}(p)}{1^{T} \boldsymbol{C}_{\boldsymbol{T}}^{-1} 1}$,

where $1=(1, \ldots, 1)$, as in Bennett et al. (2003) and Eriksen et al. (2004).

\subsection{Multiple spectral constraints}

In some particular cases, the emission spectra of various astrophysical components may be known with sufficient accuracy. Thus, it is possible to use multiple constraints (i.e., $\left.g_{1}, g_{2}, \ldots, g_{N_{\mathrm{nc}}}\right)$ for the minimization process to extract some astrophysical components and to reject some others. In practice, we can write those constraints as

$$
\begin{aligned}
& g_{1}=\boldsymbol{w}^{\boldsymbol{T}} \boldsymbol{f}_{1}=1 \\
& g_{2}=\boldsymbol{w}^{\boldsymbol{T}} \boldsymbol{f}_{2}=0
\end{aligned}
$$

$g_{N_{\mathrm{rc}}+1}=\boldsymbol{w}^{T} \boldsymbol{f}_{N_{\mathrm{rc}}+1}=0$

where $N_{\text {rc }}$ are the number of rejected components.

Remazeilles et al. (2011) have shown that two components can be constrained and simultaneously recovered. We propose here a generalization of this method to an arbitrarily large number of constraints. Defining $\mathcal{F}$ as a matrix with $N_{\text {obs }} \times\left(1+N_{\text {rc }}\right)$ elements such that

$\mathcal{F}=\left(\begin{array}{cccc}\boldsymbol{f}_{1}[1] & \boldsymbol{f}_{2}[1] & \cdots & \boldsymbol{f}_{1+N_{\mathrm{rc}}}[1] \\ \vdots & \vdots & \ddots & \vdots \\ \boldsymbol{f}_{1}\left[N_{\mathrm{obs}}\right] & \boldsymbol{f}_{2}\left[N_{\mathrm{obs}}\right] & \cdots & \boldsymbol{f}_{1+N_{\mathrm{rc}}}\left[N_{\mathrm{obs}}\right]\end{array}\right)$,

Equation (9) can be generalized to

$$
\left(\begin{array}{cc}
2 . C_{T} & -\mathcal{F} \\
\mathcal{F}^{T} & 0
\end{array}\right)\left(\begin{array}{l}
w \\
\lambda
\end{array}\right)=\left(\begin{array}{l}
0 \\
e
\end{array}\right),
$$

where $\lambda=\left(\lambda_{(1)}, \lambda_{(2)}, \cdots, \lambda_{\left(1+N_{\mathrm{rc}}\right)}\right)^{T}$ are the Lagrange multipliers and $\boldsymbol{e}=(1,0, \ldots, 0)^{T}$. The solution of the system can then be written as

$\widehat{S}_{1}(p)=\boldsymbol{e}^{\boldsymbol{T}} \boldsymbol{W}^{\boldsymbol{T}} \boldsymbol{T}(p)=\left\{\boldsymbol{e}^{T}\left(\mathcal{F}^{T} \boldsymbol{C}_{\boldsymbol{T}}^{-1} \mathcal{F}\right)^{-1} \mathcal{F}^{T} \boldsymbol{C}_{\boldsymbol{T}}^{-1}\right\} \boldsymbol{T}(p)$,

with $\mathcal{W}$ the $\left(N_{\mathrm{rc}}+1\right) \times N_{\mathrm{obs}}$ dimension matrix containing the weights extracting each constrained components. The first line of this matrix contains the weights for the extraction of the components of interest, $\boldsymbol{w}=\boldsymbol{W} \boldsymbol{e}$.

We stress that in the relation $\mathcal{F}=\mathcal{A} \boldsymbol{e}_{\boldsymbol{c}}, \boldsymbol{e}_{\boldsymbol{c}}$ is now a $N_{\mathrm{s}} \times(1+$ $N_{\text {rc }}$ ) matrix.

\section{Bias in the standard ILC estimator}

The ILC estimator as presented above is biased. This bias can have several origins:

- correlation between the "wanted" components and the other astrophysical components;

- differences between the true emission laws and the constraints used to minimize the variance. These differences can be produced by calibration uncertainties (Dick et al. 2010) or by discrepancies between the assumed emission laws and the actual ones;

- noise-induced correlations.

\subsection{Intrinsic bias}

We first characterize the bias in the ILC estimator proposed in Eq. (10), neglecting the contribution of the instrumental noise. In this situation, we can write the channel covariance matrix as

$C_{T}=\mathcal{A} C_{\mathrm{S}} \mathcal{A}^{T}$,

with $C_{\mathrm{S}}$ the $N_{\mathrm{s}} \times N_{\mathrm{s}}$ dimension covariance matrix of the astrophysical components. The $C_{\boldsymbol{T}}$ matrix has a dimension $N_{\text {obs }} \times N_{\text {obs }}$ but has a rank: $\min \left(N_{\mathrm{s}}, N_{\text {obs }}\right)$, by construction; as for $C_{\mathbf{S}}$, it has a rank $N_{\mathrm{s}}$. From here, three cases are possible for the $C_{T}$ matrix inversion:

- (1) $N_{\mathrm{s}}>N_{\text {obs }}$ : there are more physical components than channels. It is not possible to describe $C_{T}$ with an $n_{\mathrm{s}}$ dimension sub-space. The $n_{\mathrm{t}}$ dimension subspace which describes the $C_{T}$ matrix thus has no physical meaning and consequently it is not possible to extract a single component by a linear combination. The ILC approach will thus produce highly biased estimation. A solution to this problem requires more information, such as other channels of observations.

- (2) $N_{\mathrm{s}}=N_{\text {obs }}$ : there are the same number of components than channels. In this case, $\mathcal{A}$ is a square matrix. There is no ambiguity in inverting $\boldsymbol{C}_{\boldsymbol{T}}$. There exist an unbiased linear combination that allows us to extract $S_{1}$.

- (3) $N_{\mathrm{s}}<N_{\text {obs }}$ : there are more channels than astrophysical components. $C_{\boldsymbol{T}}$ has a rank $N_{\mathrm{s}}$ lower than his dimension $N_{\text {obs }}$. Consequently, this matrix is singular and cannot be directly inverted. However, we can use the pseudo inverse as defined by the singular value decomposition (SVD) of the matrix. We can thus write $\boldsymbol{C}_{T}^{-1}=\mathcal{U} \mathcal{D}^{-1} \mathcal{U}^{T}$, with $\mathcal{U}$ an orthogonal matrix and $\mathcal{D}$ a diagonal matrix containing the singular values of $C_{T}$. The $\mathcal{D}^{-1}$ matrix is obtained by taking the inverse of all singular values different from zero, and setting to zero the inverse of all singular values equal to zero. The $\mathcal{D}$ matrix is uniquely defined, however the $\mathcal{U}$ matrix is defined with $N_{\text {obs }}-N_{\mathrm{s}}$ degrees of freedom. There exist multiple linear combinations to extract the $S_{1}$ component.

When the $\mathcal{A}$ matrix is rectangular $\left(N_{\mathrm{s}}<N_{\text {obs }}\right)$, the notation $\mathcal{A}^{-1}$ refers to the left inverse of the matrix (also defined with $N_{\text {obs }}-N_{\mathrm{s}}$ degrees of freedom).

For each pixel, we can also define $\mathbf{S}^{\prime}(p)$ and $\mathbf{S}^{\prime \prime}(p)$ two vectors of dimensions $N_{\text {rc }}+1$ and $N_{\mathrm{s}}-N_{\text {rc }}-1$ respectively, that contain the constrained and un-constrained components such that

$\mathbf{S}(p)=\left(\begin{array}{c}\mathbf{S}^{\prime}(p) \\ \mathbf{S}^{\prime \prime}(p)\end{array}\right)$

For simplicity hereafter, we do not explicitly write the pixel index, $p$. 
In the following, we will focus on the case $N_{\mathrm{s}} \leq N_{\text {obs }}$. Using Eq. (16), we can express the estimator of $S_{1}$ as

$\widehat{S}_{1}=\boldsymbol{e}^{\boldsymbol{T}}\left(\boldsymbol{e}_{c}^{\boldsymbol{T}} \boldsymbol{C}_{\mathrm{S}}^{-1} \boldsymbol{e}_{c}\right)^{-1} \boldsymbol{e}_{c}^{T} \boldsymbol{C}_{\mathrm{S}}^{-1} \mathbf{S}$

To simplify the following discussions, we choose to express $\boldsymbol{C}_{\mathrm{S}}$ as a function of different sub-spaces

$C_{\mathrm{S}}=\left(\begin{array}{cc}\mathcal{E}_{0} & \mathcal{G}_{0} \\ \mathcal{G}_{0}^{T} & \mathcal{H}_{0}\end{array}\right)$

with $\mathcal{E}_{0}$ the $\left(N_{\mathrm{rc}}+1\right) \times\left(N_{\mathrm{rc}}+1\right)$ dimension sub-space associated with the constrained components, $\mathcal{H}_{0}$ the $\left(N_{\mathrm{s}}-N_{\mathrm{rc}}-1\right) \times\left(N_{\mathrm{s}}-\right.$ $\left.N_{\text {rc }}-1\right)$ dimension sub-space associated with the other components. The matrix $\mathcal{G}_{0}$ is a $\left(N_{\mathrm{rc}}+1\right) \times\left(N_{\mathrm{s}}-N_{\mathrm{rc}}-1\right)$ sub-space of $C_{\mathrm{S}}$ contains the correlation between the contained and unconstrained components.

We can rewrite the estimator of $S_{1}$, using a blocwise inversion procedure

$$
\begin{aligned}
\widehat{S}_{1} & =\boldsymbol{e}^{\boldsymbol{T}}\left(\mathbf{S}^{\prime}-\mathcal{G}_{0} \mathcal{H}_{0}^{-1} \mathbf{S}^{\prime \prime}\right) \\
& =S_{1}-\boldsymbol{e}^{\boldsymbol{T}} \mathcal{G}_{0} \mathcal{H}_{0}^{-1} \mathbf{S}^{\prime \prime}
\end{aligned}
$$

We note that the $S_{1}$ component is effectively recovered. However, we also notice the presence of a term of bias $-\boldsymbol{e}^{\boldsymbol{T}} \mathcal{G}_{0} \mathcal{H}_{0}^{-1} \mathbf{S}^{\prime \prime}$ produced by the correlation, $\mathcal{G}_{0}$, between the reconstructed component and the un-constrained components. This term of bias is composed by a linear combination of the un-constraints components and it is also function of the inverse of the covariance matrix of the un-constrained component.

This bias can be intuitively understood as the astrophysical emissions correlated with the "wanted" component, $S_{1}$, can be used to minimize the variance by removing a fraction of the "wanted" emission. In order to reduce the bias induced by such correlations, it is possible to use prior information on the spatial distribution of the contaminating astrophysical components as discussed in Sect. 5.2.

\subsection{Noise-induced bias}

We now focus on the impact of the noise on the estimation of the "wanted" component, $S_{1}$. Adding noise, the $C_{T}$ matrix can be simply written as

$C_{T}=\mathcal{A} C_{\mathrm{S}} \mathcal{A}^{T}+C_{N}$

where $C_{N}=\left\langle\boldsymbol{N} N^{T}\right\rangle$ is the $N_{\text {obs }} \times N_{\text {obs }}$ dimension covariance matrix of the instrumental noise averaged across pixels. This matrix is diagonal if the noise between different observation channels is uncorrelated. This matrix has a rank $N_{\text {obs }}$, consequently $\boldsymbol{C}_{\boldsymbol{T}}$ has also rank $N_{\text {obs }}$ and can be inverted in any case. The $C_{T}$ matrix can be projected in the space of the astrophysical components as

$\mathcal{A}^{-1} C_{T} \mathcal{A}^{-T}=C_{\mathrm{S}}+\mathcal{A}^{-1} C_{N} \mathcal{A}^{-T}$

Assuming that $C_{N}$ is a diagonal matrix (this is the case for the applications presented in this paper), $\mathcal{A}^{-1} \boldsymbol{C}_{\boldsymbol{N}} \mathcal{A}^{-\boldsymbol{T}}$ is not. The noise covariance matrix adds non-diagonal terms in $\mathcal{A}^{-1} \boldsymbol{C}_{\boldsymbol{T}} \mathcal{A}^{-T}$. As discussed in Sect. 4.1, this term will add a bias in the estimator of $\widehat{S}_{1}$ such that

$\widehat{S}_{1}=e^{T}\left(\mathcal{F}^{T} C_{T}^{-1} \mathcal{F}\right)^{-1} \mathcal{F}^{T} C_{T}^{-1} \boldsymbol{T}$
Writing $\boldsymbol{T}=\mathcal{A S}+\boldsymbol{N}$ (signal plus noise terms) Eq. (23) reads as

$$
\begin{aligned}
& \widehat{S}_{1}=\boldsymbol{e}^{\boldsymbol{T}}\left(\boldsymbol{e}_{c}^{T}\left(\boldsymbol{C}_{\mathbf{S}}+\mathcal{A}^{-1} \boldsymbol{C}_{N} \mathcal{A}^{-\boldsymbol{T}}\right)^{-1} \boldsymbol{e}_{c}\right)^{-1} \boldsymbol{e}_{c}^{T}\left(\boldsymbol{C}_{\mathbf{S}}+\mathcal{A}^{-1} \boldsymbol{C}_{N} \mathcal{A}^{-\boldsymbol{T}}\right)^{-1} \mathbf{S} \\
& +\boldsymbol{e}^{\boldsymbol{T}}\left(\boldsymbol{e}_{c}^{T}\left(\boldsymbol{C}_{\mathbf{S}}+\mathcal{A}^{-1} \boldsymbol{C}_{\boldsymbol{N}} \mathcal{A}^{-\boldsymbol{T}}\right)^{-1} \boldsymbol{e}_{c}\right)^{-1} \boldsymbol{e}_{c}^{T} \mathcal{A}^{\boldsymbol{T}}\left(\mathcal{A} \boldsymbol{C}_{\mathbf{S}} \mathcal{A}^{\boldsymbol{T}}+\boldsymbol{C}_{\boldsymbol{N}}\right)^{-1} \boldsymbol{N}
\end{aligned}
$$

We observe two terms, one depending on the astrophysical signal and the second depending on the noise contribution. To express clearly the extra-bias produced by noise, we rewrite the matrix $\mathcal{A}^{-1} \mathcal{C}_{N} \mathcal{A}^{-T}$ in the same form we used for $C_{S}$ in Eq. (19)

$$
\mathcal{A}^{-1} \boldsymbol{C}_{N} \mathcal{A}^{-\boldsymbol{T}}=\left(\begin{array}{cc}
\mathcal{E}_{N} & \mathcal{G}_{N} \\
\mathcal{G}_{N}^{T} & \mathcal{H}_{N}
\end{array}\right)
$$

and

$$
\mathcal{A}^{-1} N=\left(\begin{array}{c}
\tilde{N}^{\prime} \\
\tilde{N}^{\prime \prime}
\end{array}\right) .
$$

We can write the residual term in our estimator in the form

$$
\begin{aligned}
R_{1} & =\widehat{S}_{1}-S_{1} \\
& =\boldsymbol{e}^{\boldsymbol{T}} \tilde{\boldsymbol{N}}^{\prime}-\boldsymbol{e}^{\boldsymbol{T}}\left(\mathcal{G}_{0}+\mathcal{G}_{N}\right)\left(\mathcal{H}_{0}+\mathcal{H}_{N}\right)^{-1}\left(\mathbf{S}^{\prime \prime}+\tilde{\boldsymbol{N}}^{\prime \prime}\right) \\
& =\boldsymbol{e}^{\boldsymbol{T}} \tilde{\boldsymbol{N}}^{\prime}-\boldsymbol{e}^{\boldsymbol{T}}\left(\mathcal{G}_{0}+\mathcal{G}_{N}\right) \mathcal{H}_{0}^{-1}\left[\sum_{k=0}^{\infty}(-1)^{k}\left(\mathcal{H}_{0}^{-1} \mathcal{H}_{N}\right)^{k}\right]\left(\mathbf{S}^{\prime \prime}+\tilde{\boldsymbol{N}}^{\prime \prime}\right) .
\end{aligned}
$$

The term $\mathcal{G}_{N}$ produces extra-bias. The additional bias introduced by the noise can be reduced if we have an estimate of the noise covariance matrix, $C_{N}$. We will discuss the correction of this bias and its caveats in Sect. 5.

\section{Modified internal linear combination algorithm (MILCA)}

To reduce the bias presented in the previous section, we propose several modifications to the standard ILC estimator:

- (1) localization in pixel and spherical harmonic spaces to account for spatial spectral law variations;

- (2) modify the definition of the variance we minimize (by an action on the covariance matrix, which is equivalent to a modification of the Lagrangian of the problem), to account for noise-induced and astrophysical correlations;

- (3) add extra constraints as discussed in Sect. 3.2.

\subsection{Localization in the pixel and spherical harmonic domains}

Astrophysical components properties vary both spatially (pixel domain) and in frequency (spherical harmonic domain). For example, the CMB is homogeneous and isotropic over the sky and is dominant at a typical angular scale of about one degree. Galactic foregrounds are localized in the Galactic plane and at large angular scales with spectral laws that vary smoothly spatially. Extragalactic foregrounds are localized at small angular scales, with emission laws changing significantly from one object to another. Consequently, using a reconstruction localized both in space and frequency allows us to improve the ILC performance, by adapting the weights $w$ to the local background. This point has intensively been discussed in the literature (see e.g. Basak \& Delabrouille 2012; Bobin et al. 2013). 
We choose here to filter the observations with $N_{k}$ filters in spherical harmonic space. These filters are built from the difference between two Gaussian filters of the form $B_{l}^{\alpha}=\exp (-l(l+$ 1) $\left.\sigma_{\alpha}^{2} / 2\right)$ such that

$F_{l}^{\alpha}=B_{l}^{\alpha}-B_{l}^{\alpha+1}$

where $\sigma_{\alpha}^{2}$ increases with $\alpha$. We also impose $B_{l}^{0}=1$ and $B_{l}^{k}=0$, in order that the condition

$\sum_{\alpha}^{k-1} F_{l}^{\alpha}=1$,

is satisfied.

After filtering the observation channel maps, the weights $w$ are computed locally in predefined pixel regions. To ease the procedure these regions have been built using the properties of the HEALPix pixelization NESTED scheme (Górski et al. 2005). For each filter, these regions are defined by HEALPix pixels at a given resolution $N_{\text {side }}^{\alpha}$ such that the size of the pixel is 20 times greater than $\sigma_{\alpha+1}$ and $N_{\text {side }}^{\alpha} \leq \frac{N_{\text {side }}}{16}$, where $N_{\text {side }}$ is the native resolution of observation channel maps.

In order to ensure the continuity of the weights, $w$, at the interface between two contiguous pixel regions at the resolution $N_{\text {side }}^{\alpha}$, the map of weights at the native resolution $N_{\text {side }}$ are convolved with a Gaussian beam with a FWHM equal to the size of the HEALPix pixel at the resolution $N_{\text {side }}^{\alpha}$.

\subsection{External template regularization of the ILC solution}

In the following, we consider the possibility of using external templates to minimize the contribution of "unwanted" components, particularly those for which the electromagnetic spectrum is poorly known. For example, point-like sources and compact objects have varying electromagnetic spectra and therefore are difficult to handle with the standard ILC algorithm.

One of the main advantages of ILC methods is to only use information from a single experiment in the linear combination. We present here an approach allowing us to use external priors for the computation of the weights $w$ but not using the external templates themselves in the linear combination. To do so, we modify the data covariance matrix $C_{T}$ to include an extra term. Given "unwanted" astrophysical components, we first compute their expected contribution to the data covariance matrix and then we exacerbate this contribution to force the ILC algorithm to minimize them in the final estimate of $\widehat{S}_{1}$. In practice, we write

$C_{T}^{\prime}=C_{T}+\left(\gamma^{2}-1\right) C_{C}$,

where $C_{C}$ is a $N_{\text {obs }} \times N_{\text {obs }}$ dimension matrix containing the contribution of the "unwanted" astrophysical components to the data covariance matrix as estimated from external templates of these components. The multiplicative factor $\gamma$ takes only values higher than one and is adapted depending on the accuracy of the estimated $\boldsymbol{C}_{C}$. Writing $\boldsymbol{C}_{C}$ as

$C_{C}=\mathcal{A}\left(\begin{array}{cc}0 & 0 \\ 0 & \mathcal{H}_{C}\end{array}\right) \mathcal{A}^{T}$

we can derive the following expression for the residuals

$$
\begin{aligned}
R_{1} & =\boldsymbol{e}^{\boldsymbol{T}}\left[\tilde{\boldsymbol{N}}^{\prime}-\left(\mathcal{G}_{0}+\mathcal{G}_{N}\right)\left(\mathcal{H}_{0}+\left(\gamma^{2}-1\right) \mathcal{H}_{C}+\mathcal{H}_{N}\right)^{-1}\left(\mathbf{S}^{\prime \prime}+\tilde{N}^{\prime \prime}\right)\right] \\
& =\boldsymbol{e}^{\boldsymbol{T}}\left[\tilde{\boldsymbol{N}}^{\prime}-\left(\mathcal{G}_{0}+\mathcal{G}_{N}\right)\left(\mathcal{H}_{0}^{\prime}+\mathcal{H}_{N}\right)^{-1}\left(\mathbf{S}^{\prime \prime}+\tilde{N}^{\prime \prime}\right)\right] .
\end{aligned}
$$

As we are not constraining all astrophysical components, the $\mathcal{H}_{C}$ matrix is in general singular. Consequently, this matrix only modifies a sub-space of $\boldsymbol{H}_{0}$.

In Sect. 6.2, we present a practical application of this technique in order to reduce the CIB contamination in the reconstructed tSZ map. We observe that it leads to a reduction of the bias in the residuals proportional to the factor $\simeq \gamma^{2}$, but also to an increase in the noise.

\subsection{Reducing the noise-induced bias}

As discussed in Sect. 4.2, instrumental noise produces a bias in the estimate of $S_{1}$. To remove this bias, Vio \& Andreani (2008) proposed an unbiased estimator that can be obtained by modifying the data covariance matrix as follows

$\widetilde{C}_{T}=C_{T}^{\prime}-\widehat{C}_{N}$

If the direct subtraction of the noise in $\boldsymbol{T}$ is not possible, the estimation of the noise covariance matrix $C_{N}$ is possible. The estimate of the data covariance matrix in Eq. (33) allows us to obtain an estimate of $S_{1}$ equivalent to the one for the noiseless case, as presented in Sect. 4.1. Consequently, $\widehat{S}_{1}$ is only biased by the correlation between the "wanted", $S_{1}$, and the other components. Indeed, we obtain the following expression for the residual

$R_{1}=\widehat{S}_{1}-S_{1}=e^{T}\left[\tilde{N}^{\prime}-\mathcal{G}_{0} \mathcal{H}_{0}^{\prime-1}\left(\mathbf{S}^{\prime \prime}+\tilde{N}^{\prime \prime}\right)\right]$.

The excess of bias introduced by the noise covariance matrix has been suppressed.

However in this situation, we do no longer minimize the contribution of the noise to the data covariance matrix $C_{T}$. Furthermore, the $\widetilde{\boldsymbol{C}}_{T}$ matrix is singular for the case $N_{\mathrm{s}}<N_{\text {obs }}$. This can lead to a significant increase in the instrumental noise on $S_{1}$. To balance this effect and minimize the noise contribution, we add a regularization term to the ILC algorithm. We use the remaining $N_{\mathrm{obs}}-N_{\mathrm{s}}$ degrees of freedom in the definition of the pseudo inverse for the $\tilde{C}_{T}$ matrix.

We start by clearly identifying the subspaces of $\widetilde{C_{T}}$ that are constrained by the ILC constraints and the variance minimization respectively. The ILC constraints act on a $N_{\text {rc }}+1$ dimension subspace, then the minimization of the variance act on a $N_{\mathrm{s}}-N_{\mathrm{rc}}-1$ dimension subspace. We still have an $N_{\mathrm{obs}}-N_{\mathrm{s}}$ dimension subspace to minimize the instrumental noise contribution.

\subsubsection{Constrained-components subspace}

In order to isolate the subspace associated with the ILC constraints, we propose to perform a transformation of the form

$$
\begin{aligned}
\widetilde{\boldsymbol{C}_{\boldsymbol{T}}} & =\boldsymbol{C}_{\boldsymbol{T}}^{\prime}-\boldsymbol{C}_{\boldsymbol{N}}-\alpha \mathcal{F}\left(\mathcal{F}^{\boldsymbol{T}}\left(\boldsymbol{C}_{\boldsymbol{T}}^{\prime}-\boldsymbol{C}_{N}\right)^{-1} \mathcal{F}\right)^{-1} \mathcal{F}^{\boldsymbol{T}} \\
& =\boldsymbol{C}_{\boldsymbol{T}}^{\prime}-\boldsymbol{C}_{\boldsymbol{N}}-\alpha \mathcal{A} \boldsymbol{e}_{c}\left(\mathcal{E}_{0}-\mathcal{G}_{0} \mathcal{H}_{0}^{\prime-1} \mathcal{G}_{0}^{\boldsymbol{T}}\right) \boldsymbol{e}_{c}^{\boldsymbol{T}} \mathcal{A}^{\boldsymbol{T}} \\
& =\boldsymbol{C}_{\boldsymbol{T}}^{\prime}-\boldsymbol{C}_{N}-\alpha \mathcal{A} \boldsymbol{e}_{c} \widehat{\mathcal{E}}_{0} \boldsymbol{e}_{c}^{\boldsymbol{T}} \mathcal{A}^{\boldsymbol{T}}
\end{aligned}
$$

with $\alpha$ the fraction of variance from the constrained components to be subtracted. For $\alpha=1$ the $\widetilde{C_{T}}$ matrix has a rank $N_{\mathrm{s}}-N_{\mathrm{rc}}-1$, the $C_{T}^{\prime}-C_{N}$ matrix having a rank $N_{\mathrm{s}}$. We subtract $C_{N}$ because we want to consider the variance of the astrophysical signal without noise. $\widehat{\mathcal{E}}_{0}=\mathcal{E}_{0}-\mathcal{G}_{0} \mathcal{H}_{0}^{\prime-1} \mathcal{G}_{0}^{\boldsymbol{T}}$ is the estimate of the covariance matrix of the constrained components for the standard ILC algorithm. We observe the contribution of the intrinsic bias term 
$-\mathcal{G}_{0} \mathcal{H}_{0}^{\prime-1} \mathcal{G}_{0}^{\boldsymbol{T}}$. Indeed, the variance of the reconstructed component reads as

$$
\begin{aligned}
V_{S_{1}} & =\boldsymbol{w}^{\boldsymbol{T}}\left(\boldsymbol{C}_{\boldsymbol{T}}^{\prime}-C_{N}\right) \boldsymbol{w} \\
& =\boldsymbol{e}^{\boldsymbol{T}}\left(\mathcal{F}^{\boldsymbol{T}}\left(\boldsymbol{C}_{T}^{\prime}-C_{N}\right)^{-1} \mathcal{F}\right)^{-1} \boldsymbol{e} \\
& =\boldsymbol{e}^{\boldsymbol{T}} \widehat{\mathcal{E}}_{0} \boldsymbol{e}
\end{aligned}
$$

Writing the data covariance matrix as

$\widetilde{\boldsymbol{C}_{T}}=\mathcal{A}\left(\boldsymbol{C}_{\mathbf{S}}^{\prime}-\alpha \boldsymbol{e}_{c} \widehat{\mathcal{E}}_{0} \boldsymbol{e}_{c}^{T}\right) \mathcal{A}^{T}$,

and we derive

$$
\begin{aligned}
\widehat{S}_{1}=\boldsymbol{e}^{T}\left[\boldsymbol{e}_{c}^{T}\left(\boldsymbol{C}_{\mathrm{S}}^{\prime}-\alpha \boldsymbol{e}_{c} \widehat{\mathcal{E}}_{0} \boldsymbol{e}_{c}^{T}\right)^{-1} \boldsymbol{e}_{c}\right]^{-1} \\
\\
\quad \times \boldsymbol{e}_{c}^{\boldsymbol{T}}\left(\boldsymbol{C}_{\mathrm{S}}^{\prime}-\alpha \boldsymbol{e}_{c} \widehat{\mathcal{E}}_{0} \boldsymbol{e}_{c}^{\boldsymbol{T}}\right)^{-1}\left(\mathbf{S}+\mathcal{A}^{-1} \boldsymbol{N}\right),
\end{aligned}
$$

the $C_{\mathrm{S}}^{\prime}-\alpha \boldsymbol{e}_{c} \widehat{\mathcal{E}}_{0} \boldsymbol{e}_{c}^{\boldsymbol{T}}$ matrix can be rewritten in the following form

$C_{\mathrm{S}}^{\prime}-\alpha \boldsymbol{e}_{c} \widehat{\mathcal{E}}_{0} \boldsymbol{e}_{c}^{T}=\left(\begin{array}{cc}\mathcal{E}_{0}-\alpha \widehat{\mathcal{E}}_{0} & \mathcal{G}_{0} \\ \mathcal{G}_{0}^{T} & \mathcal{H}_{0}^{\prime}\end{array}\right)$.

Consequently, the residual, $R_{1}$, in Eq. (34) does not depend on $\mathcal{E}_{0}$ and consequently do not depend on the parameter $\alpha$.

Indeed, the $\mathcal{E}_{0}$ sub-space is constrained, its modification will not produce any modification on $\widehat{S}_{1}$. We can deduce that the weights $\boldsymbol{w}$ are invariant under this transformation. So we select the value $\alpha=1$ for which $\tilde{C_{T}}$ becomes $N_{\mathrm{rc}}+1$ singular.

To estimate the value of $N_{\mathrm{s}}-N_{\mathrm{rc}}-1$ components, we compute the number of eigenvalues of the $\tilde{C_{T}}$ matrix which are significantly greater than 0 . It is important to note that we also have an estimate of $N_{\text {obs }}-N_{\mathrm{s}}$ giving the remaining degrees of freedom that can be used to reduce the noise contribution in $\widehat{S}_{1}$. In the case $N_{\mathrm{s}}<N_{\text {obs }}$, the pseudo inverse of $\tilde{\boldsymbol{C}}_{\boldsymbol{T}}$ is defined with $N_{\text {obs }}-N_{\mathrm{s}}$ degrees of freedom as explained in the Sect. 4.2.

\subsubsection{Minimizing the noise variance}

To reduce the noise contribution in the final estimate of $\widehat{S}_{1}$, we also minimize simultaneously the variance of the noise term

$V_{N}=\boldsymbol{w}^{T} \boldsymbol{C}_{N} \boldsymbol{w}$

This can be performed by modifying the lowest eigenvalues of the $\tilde{C}_{T}$ matrix, assuming that they are not associated to astrophysical emissions. We search for the value of the eigenvalues $D_{j}$ of $\tilde{\boldsymbol{C}}_{\boldsymbol{T}}=\mathcal{U} \mathcal{D} \mathcal{U}^{T}$ that minimize $V_{N}$. The minimization is performed numerically and iteratively. It is important to notice that $V_{N}$ might have several extrema. Consequently, we use the first and second derivative of the variance to find the minimum of $V_{N}$.

$\frac{\partial V_{N}}{\partial D_{j}}=\boldsymbol{e}^{\boldsymbol{T}}\left(\frac{\partial \boldsymbol{W}^{\boldsymbol{T}}}{\partial D_{j}} \boldsymbol{C}_{N} \boldsymbol{W}+\boldsymbol{W}^{T} C_{N} \frac{\partial \mathcal{W}}{\partial D_{j}}\right) \boldsymbol{e}=0$

The derivative of the weight matrix $\boldsymbol{W}$ can be written as

$$
\frac{\partial^{k} \boldsymbol{W}}{\partial D_{j}^{k}}=D_{j}^{-k}\left[\left(\boldsymbol{W} \mathcal{F}^{\boldsymbol{T}}-\mathcal{I}\right) \mathcal{U} \mathcal{J} \mathcal{U}^{T}\right]^{k} \boldsymbol{W},
$$

with $\mathcal{J}=\frac{\partial \mathcal{D}}{\partial D_{j}}$

\subsection{Uncertainty estimation and propagation}

As the MILCA procedure is fully linear, it is possible to propagate analytically the statistical uncertainties induced by the instrumental noise. However, we use spatial and harmonic filters that are correlated (see Sect. 5.1). Consequently, the propagation of uncertainties is possible, but very costly. In the most generic case dealing with inhomogeneous correlated noise, noise propagation can be performed using Monte-Carlo simulations (see e.g. Planck Collaboration 2013b, for a description of such a procedure).

The estimation of foreground contamination is possible if we have templates and estimates of the SEDs. In this case, it is possible to propagate the template trough the same linear combination than the one applied to the data to provide an estimation of the contamination. Such approach has been used to estimate the contamination of the thermal dust, the CIB and point sources in the Planck Compton-y parameter maps in Planck Collaboration (20131).

We stress that one of the advantages of using a linear combination approach is to be able to easily estimate uncertainties and biases in the reconstructed signal.

\subsection{Summary of the main MILCA steps}

The main steps of MILCA are the following:

(1) Filter the original data to ensure space and frequency localization.

(2) Use the $N_{\text {rc }}+1$ constraints on the spectral emission laws of the known components.

(3) Subtract the instrumental noise contribution to the covariance matrix.

(4) Minimize of the variance of $S_{1}$ in the sub-space associated to the $N_{\mathrm{s}}-N_{\mathrm{rc}}-1$ non-constrained astrophysical components.

(5) Use the extra $N_{\text {obs }}-N_{\mathrm{s}}$ degrees of freedom to reduce the instrumental noise contribution.

The final MILCA estimate of the "wanted" component reads as

$\widehat{S}_{\mathrm{c}}=\boldsymbol{e}^{T}\left(\mathcal{F}^{T} \tilde{\boldsymbol{C}}_{\boldsymbol{T}}^{-1} \mathcal{F}\right)^{-1} \mathcal{F}^{T} \tilde{\boldsymbol{C}}_{\boldsymbol{T}}^{-1} \boldsymbol{T}$

For the case $N_{\text {rc }}+1=N_{\mathrm{s}}$, MILCA provides the same result as a maximum likelihood approach

$\widehat{\mathbf{S}}=\left(\mathcal{A}^{T} C_{N}^{-1} \mathcal{A}\right)^{-1} \mathcal{A}^{T} C_{N}^{-1} T$

The $w$ vector cannot be constrained using the minimization of the variance of components. The last $N_{\mathrm{obs}}-N_{\mathrm{s}}$ degrees of freedom are constrained by the minimization of the instrumental noise, as it is the case for a maximum likelihood method.

\section{6. $\mathrm{tSZ}$ reconstruction with MILCA}

We present here an application of the MILCA algorithm to the reconstruction of the $\mathrm{tSZ}$ effect. We first focus on the two main MILCA improvements to the ILC algorithm presented in Sect. 5. Then, we consider the extraction of a full-sky tSZ map using the Planck full-sky simulations presented in Sect. 2. We consider two constrained components, tSZ and CMB. Two degrees of freedom are used to minimize the variance of the unconstrained components and the remaining two are used to minimize the variance of the noise. The extraction of the tSZ effect has been performed at an effective resolution of $10 \mathrm{arcmin}$. 


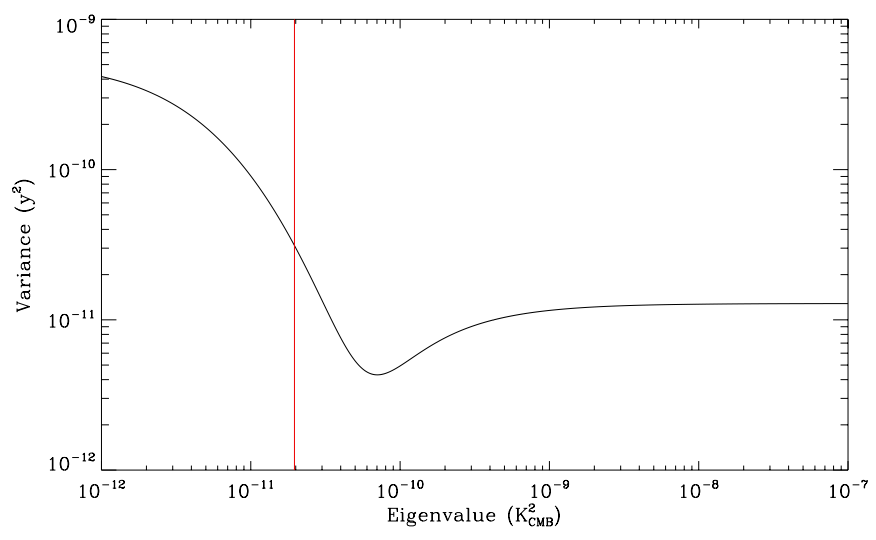

Fig. 2. Variation of the noise level in the reconstructed $y$-map as a function of the value of the modified eigenvalue in the data covariance matrix. The black line shows the noise level in the $y$-map, the red vertical line indicates the original value of the eigenvalue before modification.

\subsection{Noise variance minimization}

As discussed in Sect. 5.3.2, it is possible to reduce the noise contamination in the reconstructed map by modifying the lowest eigenvalues, $D_{j}$, of $\tilde{\boldsymbol{C}}_{T}$ to minimize $V_{N}$. For illustration purposes, we concentrate here on the lowest eigenvalue, $D_{j}$, of $\tilde{\boldsymbol{C}}_{T}$ on $V_{N}$, that we vary over five orders of magnitudes with respect to its original value. For each of these values, we compute the noise level in the reconstructed tSZ $y$-map that is shown as a solid black line in Fig. 2. In this case, the original value for the lowest eigenvalue of $\boldsymbol{C}_{\boldsymbol{T}}$ (vertical red line in Fig.2) leads to ten times larger noise level than the optimal solution that minimize the noise variance.

Furthermore, for low values of $D_{j}$ an increase in the level of noise is observed since in this case $C_{T}$ nearly singular. This produces high absolute values for $\boldsymbol{w}$, and thus a higher rms noise residual (computed following Eq. (40)). For high $D_{j}$, we converge to a rank-reduction for $C_{T}\left(D_{j}\right.$ approaches $\left.\infty\right)$, and this solution leads to a level of noise two times higher than the optimal MILCA solution.

\subsection{Minimization of the clustered CIB contamination using prior information}

We discuss now how we can use MILCA to reduce the clustered CIB contamination in the reconstructed tSZ map. Reducing clustered CIB contamination is a major issue for tSZ analysis, because the CIB is, on the one hand, correlated with the tSZ emission (Addison et al. 2012) and on the other hand, it is a diffuse emission that cannot be masked in tSZ maps.

The clustered CIB component cannot be described with a single template and an SED; furthermore, it is only partially correlated from one frequency to another (Planck Collaboration 2011f). Indeed, the CIB is produced by the IR emission from extragalactic sources at different redshifts and their observed emission law is highly dependent on the redshift. Consequently, different frequency bands are not sensitive to the same sources, according to their redshifts.

We thus cannot apply constraints on the SED, in the $\mathcal{F}$ matrix, in order to reduce the clustered CIB contamination. By contrast, we can use the approach proposed in Sect. 5.2 by modifying the data covariance matrix. The covariance matrix of the clustered CIB emission needs to account for the CIB SED and

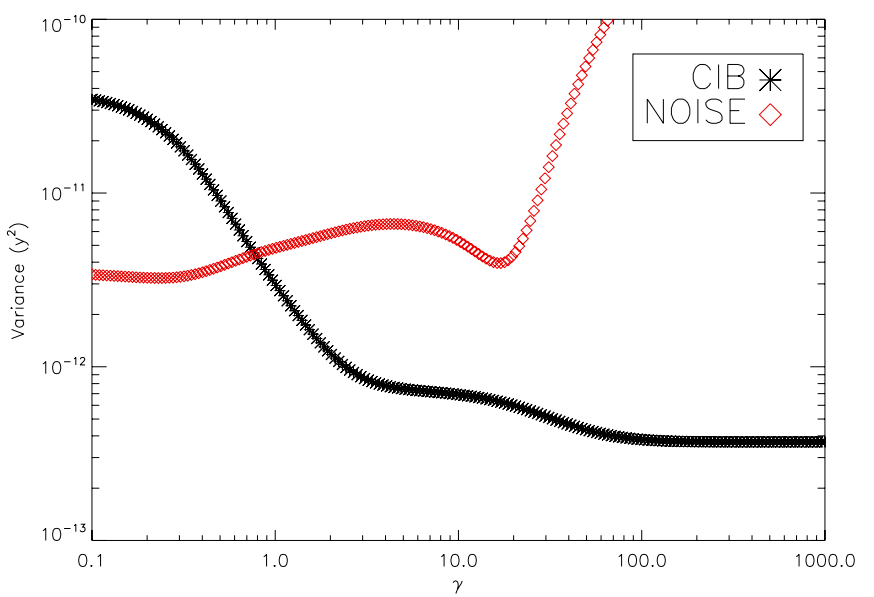

Fig. 3. Evolution of the clustered CIB contamination (black stars) and the noise level (red diamonds) as a function of $\gamma$. Both contributions (clustered CIB and noise) are presented in units of variance in the reconstructed tSZ Compton- $y$ parameter map.

for partial correlation between frequencies and it can be inferred from models or from the data themselves.

In Fig. 3, we present the variation of the clustered CIB contamination and the reconstructed tSZ map noise level as a function of the parameter $\gamma$ in Eq. (30). $\gamma<1$ corresponds to the case where we do not account for the clustered CIB and it leads to strong clustered CIB contamination. As expected, the CIB contamination decreases with as $\gamma$ increases. However, as the clustered CIB contamination decreases the noise level increases. A compromise between noise and clustered CIB contamination can be found for $\gamma$ in the range between 3 and 30, with an optimal result for $\gamma=17$, as we observe that the noise level is almost constant in this range and the clustered CIB contamination decreases with a slope $\simeq \gamma^{2}$. It is important to notice that for $\gamma=1$, the clustered CIB contamination is already reduced by one order of magnitude in variance with respect to the standard ILC algorithm. On the real data such a compromise can also be found using estimates of both the noise and clustered CIB covariance matrices. At high values of $\gamma$, we observe that the clustered CIB contamination becomes constant. This behavior is produced by the partial correlation of the clusterd CIB across frequencies. Indeed, as the CIB emission is not fully correlated between frequencies; it can not be totally removed.

\subsection{Characterization of noise level and bias on fullsky simulations}

We present in Fig. 4 the reconstructed tSZ map and the residual map in a small patch of 200 by 200 arcmin centered on the well-known pair of clusters A399-A401 (Planck Collaboration 2013e). This example illustrates the ability of MILCA to properly deal with complex tSZ systems like mergers.

In a more quantitative way, we present in Fig. 5 the histogram of the reconstructed MILCA tSZ map for all pixels located inside a radius of 15 arcmin from the 1743 clusters of the MCXC catalog (Piffaretti et al. 2011). In the top panel, we present the main contributions to the reconstructed tSZ map: tSZ emission (black), noise (green), and other astrophysical components (blue). For comparison, we also plot the input tSZ signal (red). We observe that the reconstructed tSZ map is dominated by the tSZ signal. In the bottom panel, we represent the histogram of the residual map (same color scheme as 

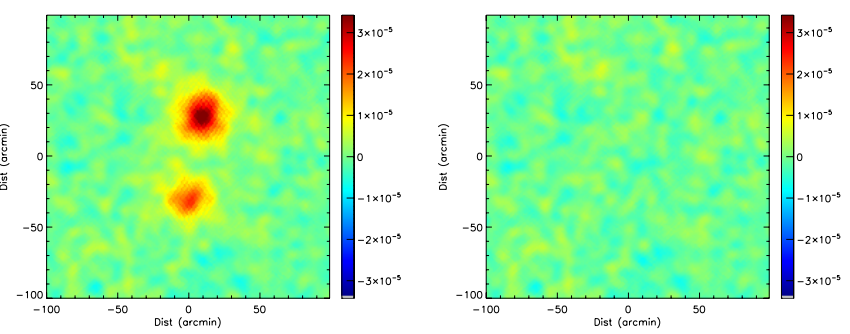

Fig. 4. Left panel: reconstruction of a tSZ $y$-map from simulated Planck data using the MILCA approach. Right panel: residual map after subtraction of the input tSZ signal.
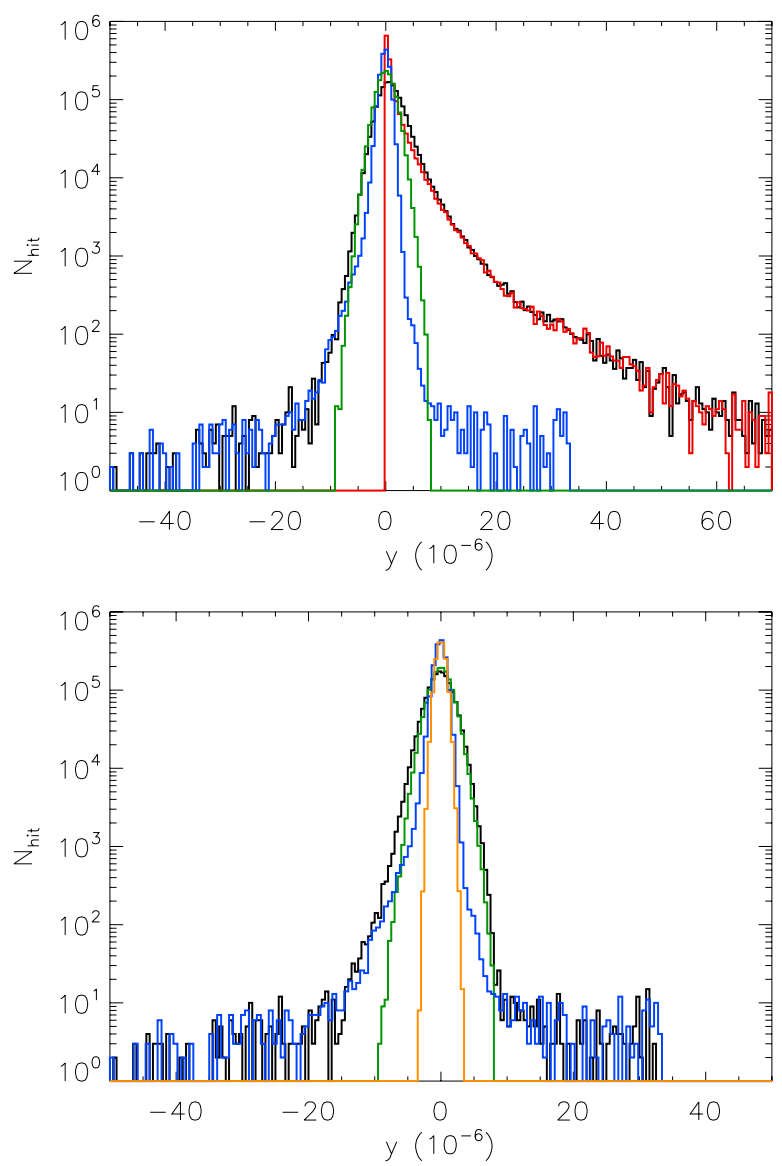

Fig. 5. Top: histograms for the MILCA tSZ map (black), for the tSZ input map (red), for the noise (green) and the contamination by other components (in blue). Bottom: histograms for the residuals emission (black), for the noise (red), for the contamination by all other components (blue) and for the CIB component contribution (green).

before). The residual map is dominated by instrumental noise which contributes to the residual $\mathrm{rms}$ at a level of $1.7 \times 10^{-6}$ (Compton parameter units), then, the CIB contributes to a level of $0.7 \times 10^{-6}$, and all other components have a total contribution of about $1.0 \times 10^{-6}$.

Notice in Fig. 5 that most of the residuals by other astrophysical components (in blue) appear as a negative bias, they are mainly produced by radio sources. Indeed, by contrast to the tSZ effect, which increases with frequency, radio source emission decreases with frequency. Consequently, when extracting the tSZ signal, radio emission generally appears as negative signal.

An extreme case of such a bias is discussed in Sect. 6.4. We also observe weak contamination by IR point sources which
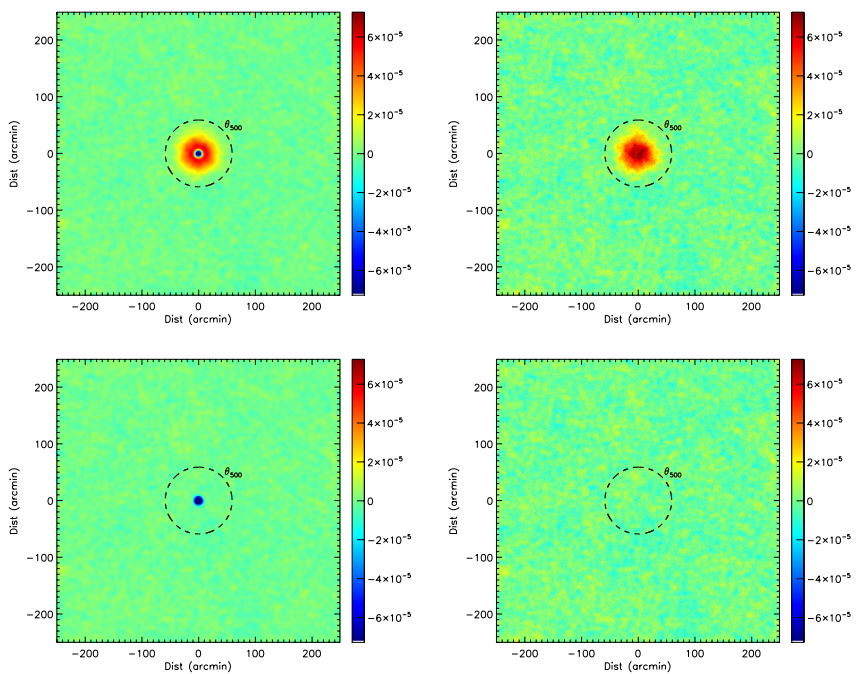

Fig. 6. Reconstructed $y$-map (top) and residuals (bottom) for the Planck simulated data using a standard ILC adapted to the tSZ effect (left panel) and using MILCA with three constrained components (right panel).

appear as positive bias in the reconstructed map. Other astrophysical emissions marginally contribute to the reconstructed signal.

An example of the application of MILCA to real Planck data is presented in Sect. 6.5.

\subsection{Empirical extra constraints for $t S Z$ extraction}

We present an application of MILCA to an extreme case of radio-loud AGN contamination for a simulated Perseus-like cluster. This particular cluster is well known to be very extended over the sky and to host a radio-loud AGN (Brunzendorf \& Meusinger 1999). The AGN emission makes the extraction of the tSZ signal for such kind of systems quite complex. However, as the tSZ signal is extended, it is possible to separate the two emissions (from the cluster and from the AGN).

Figure 6 presents the comparison between the tSZ $y$-maps (top) and residuals obtained from a standard ILC adapted to the tSZ effect (left panel) and MILCA (right panel) using three constraints to keep the tSZ signal but removing the CMB and the central radio-loud AGN contamination. We use an estimate of the AGN SED obtained directly from the data themselves. For the standard ILC case, we observe a strong contamination by the AGN emission (with an amplitude of $-9 \times 10^{-5}$ in Compton parameter units at the center of the AGN). In this case, the tSZ effect and the AGN radio emission are strongly spatially correlated over the sky. This leads to the bias discussed in Sect. 4.1, when a correlated component is used to clean the tSZ effect contribution. When considering an extra constraint, we are able to recover the tSZ signal with a much weaker bias from the AGN (an amplitude of about $-0.5 \times 10^{-6}$ ). This extra constraint presents however some drawbacks, such as an increase in the noise level (in this case by a factor of 1.5) and/or stronger bias from other astrophysical un-constrained components.

The ability of MILCA to reduce such contamination is directly related to the accuracy of the estimated SED used for the extra constraint. In order to quantify this, we performed simulations of the reconstruction but with an extra constraint applied on a noisy SED for the AGN

$f_{i}=f^{\mathrm{AGN}}\left(1+\sigma X_{i}\right)$ 

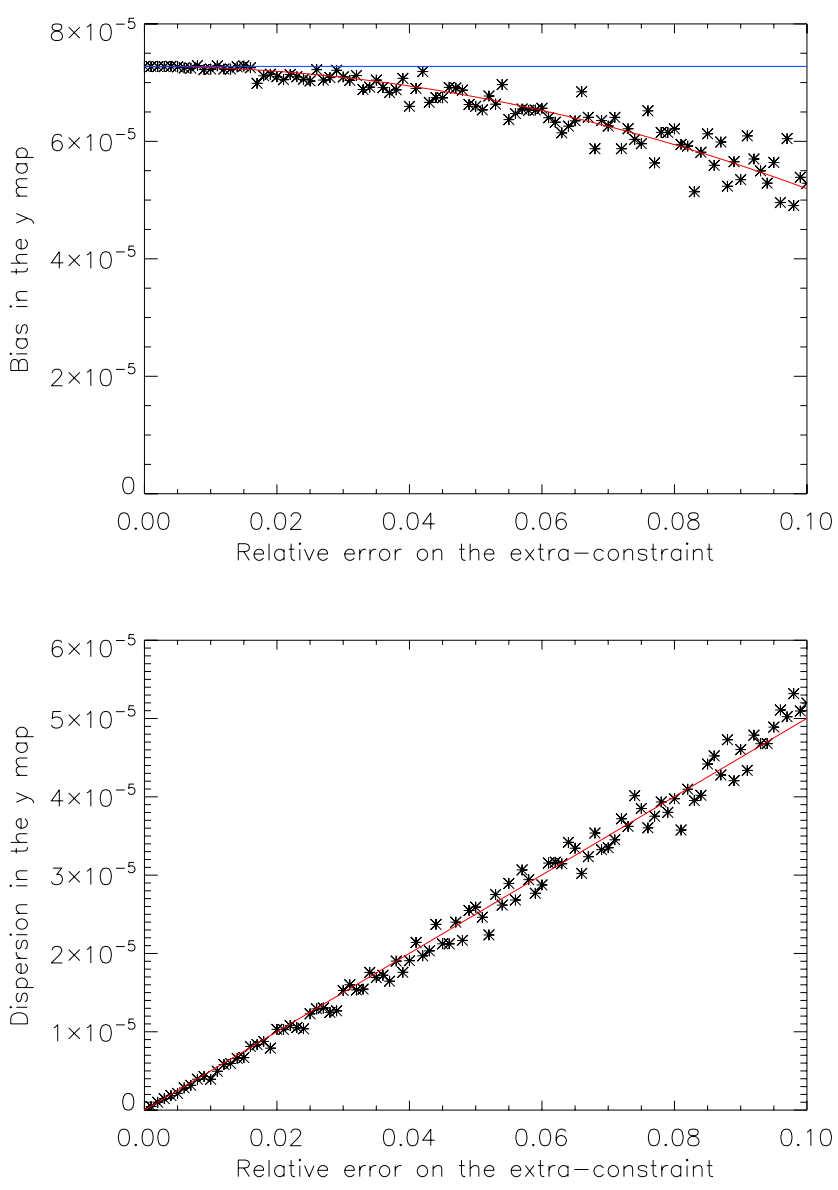

Fig. 7. Top panel: bias induced in the MILCA reconstruction when using a noisy SED as extra constraint. Black points are the mean from the 200 simulations for each value of the relative error, the red line represents the best-fit for the bias term and the blue line is the expected value in an unbiased case. Bottom panel: extra noise induced in the MILCA reconstruction when using a noisy SED as extra constraint. Black points are computed from the 200 simulation for each value of the relative error and the red line represents the best-fit for the noise term. The contamination are presented in units of Compton parameter for the central pixel of the reconstructed map for which we expect the maximum contamination.

where $f^{\mathrm{AGN}}$ is the $\mathrm{AGN}$ simulated $\mathrm{SED}, X_{i}$ is a random variable following a standard normal distribution and $\sigma$ is the relative error on the AGN SED. We performed 200 simulations of reconstruction for a grid of 100 values of $\sigma$. A global bias is computed by averaging the 200 reconstructions and the noise rms is obtained from the standard deviation of the 200 reconstructions. Figure 7 shows the bias and the extra noise induced on the reconstruction by a noisy SED. The bias is proportional to the square of the relative error, $\sigma$, while the extra noise is directly proportional to $\sigma$. This relation allows us to propagate both bias and noise from the used SED to the final reconstructed map. We stress that the calibration of this relation depends on both the AGN amplitude and the local background of the cluster. Consequently, such estimation of the noise and bias induced by a noisy SED have to be computed for each specific case.

\subsection{Extraction of the $t S Z$ effect with MILCA on real data}

For illustration in Fig. 8, we present tSZ effect reconstructed MILCA (left) maps using the Planck public data for very

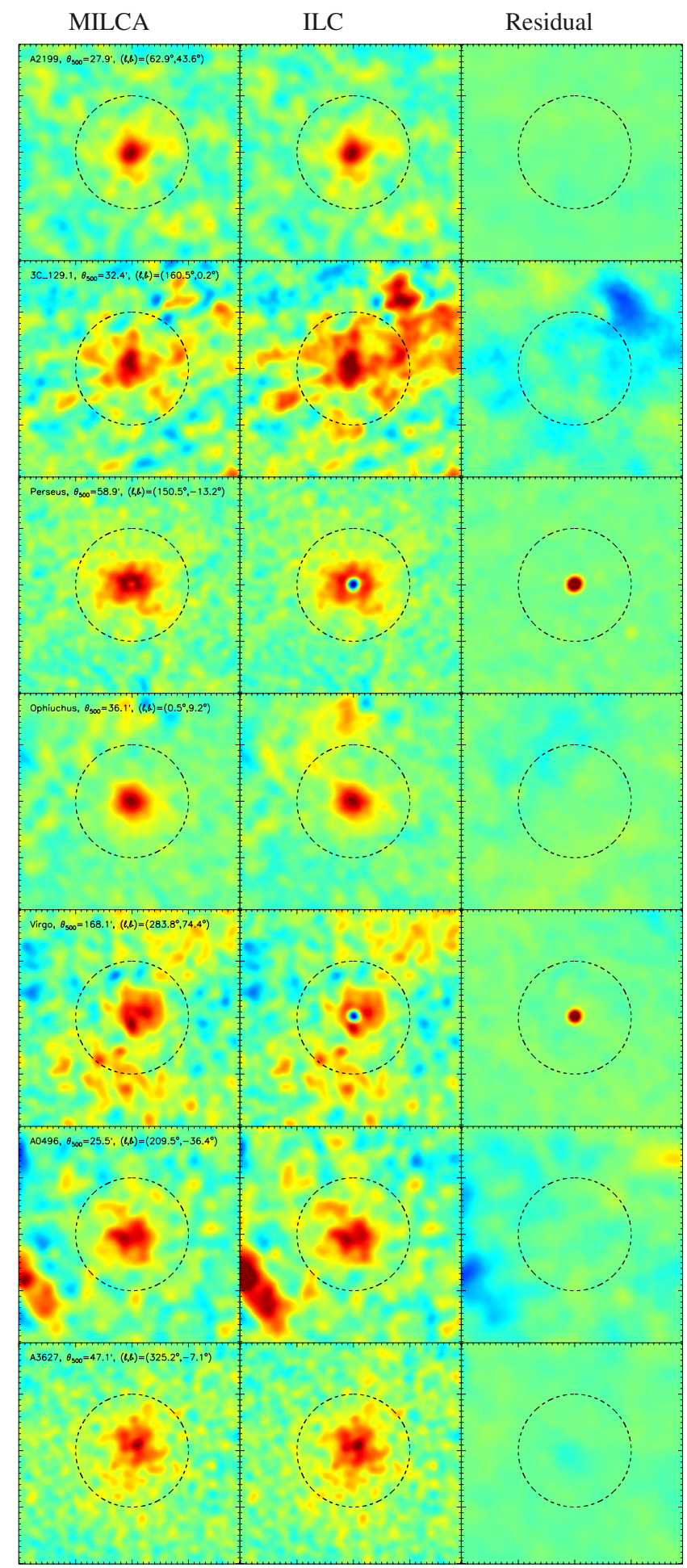

Fig. 8. From left to right: tSZ reconstructed $y$-map with MILCA from Planck public data, with a tSZ effect adapted standard ILC and the difference between the 2 reconstructions. Each row represents the reconstructions for a particular cluster in a FOV of $4 \times R_{500}$, with the dashed circle representing the $R_{500}$ aperture. For each cluster the three maps are displayed with the same color scale. All maps are presented at a resolution of 7.18' FWHM except the Virgo maps at 30' FWHM.

extended clusters over the sky (see also Planck Collaboration $2013 \mathrm{~m}$ ). We also present the comparison with a standard ILC reconstruction (middle). These figures illustrate the improvement provided by MILCA for the reconstruction of tSZ $y$-maps, not only on simulations, but also on real datasets. 
For some clusters, such as A2199, Ophiucus, and A3627, MILCA and a standard ILC adapted to tSZ extraction produce very similar results. Indeed, for these clusters the foreground contamination is low and not correlated with the tSZ component.

However in the case of AGN contaminated clusters, such as Perseus and Virgo, we observe a clear contamination in the standard ILC maps that is significantly reduced in the MILCA maps. For the Perseus galaxy cluster, we observe similar results than the ones obtained on simulated datasets in Sect. 6.4. The use of an extra constraint allows MILCA to reduce the contamination by radio-loud AGNs, which are correlated to the tSZ effect in the cluster.

For the 3C 129.1 cluster, we observe in the standard ILC map, a strong contamination around the $\mathrm{tSZ}$ emission. This contamination is mainly produced by thermal dust residuals. This cluster is located in the Galactic plane. Consequently, there is a strong contamination by dust in this area. However, the MILCA maps are less contaminated by the thermal dust emission. This reduction of the contamination by thermal dust is mainly due to the correction of the noise-induced bias.

Finally for A0496, we observe a reduction of the contamination in MILCA map but we still observe contamination by an IR point source. This IR point source is very faint with respect to the thermal dust background. Consequently, it is difficult to extract the SED of this source, reducing the ability of MILCA to minimize the source contamination in the reconstructed tSZ map.

\section{CO emission reconstruction using the Planck bolometer maps}

We present an original application of MILCA for reconstruction of $\mathrm{CO}$ emission maps for the first three $\mathrm{CO}$ transitions $J=1-$ $0, J=2-1$, and $J=3-2$ using the spectral mismatch between the bolometers within a single Planck channel as discussed in Planck Collaboration (2013h). We discuss here the methodology and performance of the algorithm using the simulations of the Planck $100 \mathrm{GHz}$ channel presented in Sect. 2. We consider four intensity maps constructed by combining the maps of two bolometers for each of the 4 PSB pairs.

Assuming bolometer maps are calibrated in CMB temperature units we can compute the bandpass mismatch for different astrophysical signals. For $\mathrm{CMB}$, we expect no bandpass mismatch within the calibration uncertainties (below 1\%). For other astrophysical components with a continuous emission spectrum, the variation of transmission in the different bolometers is very small (about $1 \%$ at $100 \mathrm{GHz}$ ). However, for molecular line associated emission, such as $\mathrm{CO}$, the transmission variations from one bolometer to another can reach up to $20 \%$ (Planck Collaboration 2013h). We use this variation of transmission among bolometers to produce $\mathrm{CO}$ maps using MILCA. Formally speaking, bandpass mismatch can be accounted for by assuming a bolometer dependent mixing matrix (rather than one depending on observation frequency) and then component separation algorithms can be applied. However, there are several problems with this approach. First, spectral bandpass mismatch is very sensitive to the SED of the astrophysical component and it might be difficult to estimate for those astrophysical components for which the SED vary spatially. Second, the signal-tonoise ratio is low with respect to conventional problems based on channel maps. MILCA is well-adapted for this particular problem as it allows us on the one hand to only use the bandpass mismatch for those components for which is well known (mainly
CMB and the "wanted" component) and on the other hand to minimize the noise contribution in the final recovered map.

We performed 10000 simulations of the Planck bolometer maps using different simulated bandpasses based on the $\mathrm{CO}$ unit conversion coefficients values and uncertainties presented in Planck Collaboration (2013h). Figure 9 shows the comparison between the reconstructed $\mathrm{CO}$ emission at $100 \mathrm{GHz}$ for an ILC algorithm spectrally adapted to CO (left) and for MILCA (right) for one these simulations. The top panel shows the reconstructed $\mathrm{CO}$ map and the bottom panel the residuals in $\mathrm{K} \mathrm{km} \mathrm{s}^{-1}$ units. We observe that the $\mathrm{CO}$ emission is well reconstructed with MILCA but in the inner Galactic plane where we observe some residual contamination. However, the ILC reconstructed map shows strong contamination by the CMB and the Galactic diffuse emission, and a higher noise level. In Table 1, we summarize the contamination in terms of contribution to the variance of the CO reconstructed map for MILCA and for the CO adapted ILC. We notice that MILCA allows us to produce $\mathrm{CO}$ maps with a level of contamination 5 times lower than a CO adapted ILC. At a resolution of $10 \mathrm{arcmin}$, the residual of the MILCA reconstructed CO map is dominated by the noise contribution, $6.06 \mathrm{~K} \mathrm{~km} \mathrm{~s}^{-1}$, other components contribute only for $0.31 \mathrm{~K} \mathrm{~km} \mathrm{~s}^{-1}$. For comparison, in the ILC CO map the noise contribution is about $7.55 \mathrm{~K} \mathrm{~km} \mathrm{~s}^{-1}$, and the contamination by other components reaches $5.53 \mathrm{~K} \mathrm{~km} \mathrm{~s}^{-1}$. We also notice that in the case of a MILCA reconstruction at 30 arcmin FWHM, the standard deviation of the map $\left(9.35 \mathrm{~K} \mathrm{~km} \mathrm{~s}^{-1}\right)$ is completely dominated by the $\mathrm{CO}$ emission $\left(9.11 \mathrm{~K} \mathrm{~km} \mathrm{~s}^{-1}\right)$ indicating the high level of purity of the map. For the ILC CO reconstructed map the standard deviation of the total map is $5.94 \mathrm{~K} \mathrm{~km} \mathrm{~s}^{-1}$, which is below the $\mathrm{CO}$ only contribution $\left(9.11 \mathrm{~K} \mathrm{~km} \mathrm{~s}^{-1}\right)$. This illustrates the strong bias in the ILC map for which the thermal dust emission is used to remove $\mathrm{CO}$ emission and then to reduce the total variance of the map.

Figure 10 presents the contamination level in the CO map for the 10000 simulations for the ILC and the MILCA reconstructions. We represent on these figures the contamination by the CMB, the thermal dust + CIB, the tSZ effect and the synchrotron + free-free + radio point sources and by the noise.

For CMB, the contamination in MILCA map is equal to zero at the calibration uncertainties level, but in the ILC map we observe a strong contamination by the $\mathrm{CMB}$, which amounts to $2.5 \mathrm{~K} \mathrm{~km} \mathrm{~s}^{-1}$ in average in terms of the standard deviation. This contamination is mainly due to the correlation between the $\mathrm{CO}$ emission and the thermal dust emission. Indeed, thermal dust emission has a continuous emission law over frequency, and has thus almost the same spectral response than the CMB in the difference bolometers. Consequently, we observe similar residuals for all contaminating astrophysical emission.

\section{Conclusion}

Component separation techniques are now a major ingredient in the scientific exploitation of multichannel and multicomponent datasets such as those of the CMB satellite experiments WMAP and Planck.

In this paper, we have presented the modified internal linear algorithm (MILCA) specially developed for the Planck data. MILCA generalizes the standard ILC algorithm, generally devoted to CMB emission extraction, to any astrophysical component with a known emission law. In practice, MILCA can be used to extract an arbitrary number of astrophysical emissions rejecting the contribution from others for which the emission law is also known. MILCA has been optimized in various 

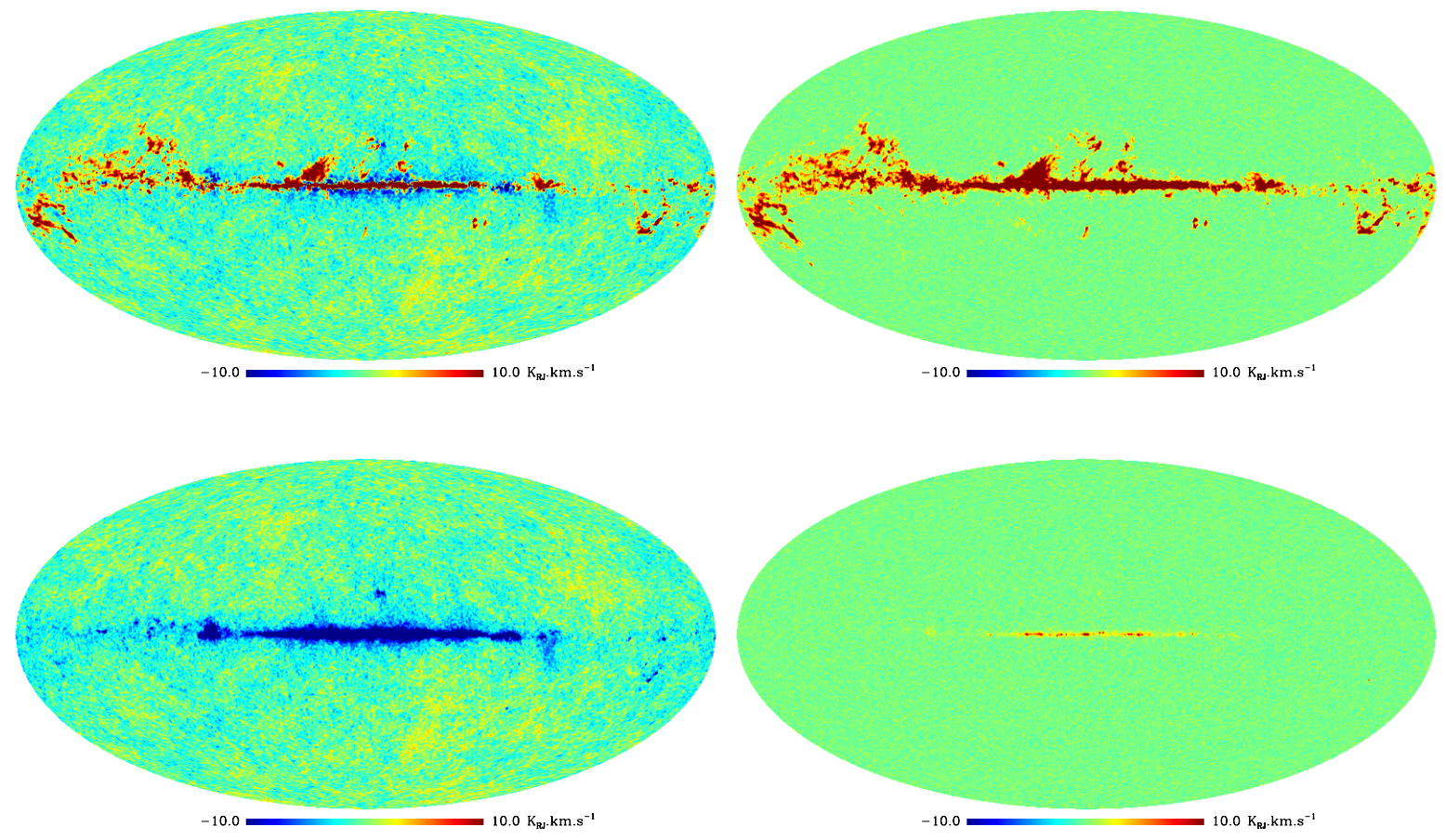

Fig. 9. Reconstructed CO emission map (top) and residuals (bottom) for the CO-adapted ILC algorithm (left) and for MILCA (right). The maps are presented at a resolution of 30 arcmin to reduce the noise contribution in the maps.

Table 1. Contribution to the total standard deviation of the reconstructed $\mathrm{CO}$ emission map in units of $\mathrm{K} \mathrm{km} \mathrm{s}^{-1}$.

\begin{tabular}{lcc}
\hline \hline Maps & CO ILC $\left(10^{\prime}\right)$ & CO MILCA $\left(10^{\prime}\right)$ \\
\hline Total & 10.43 & 12.05 \\
CO & 10.16 & 10.16 \\
Residuals & 9.36 & 6.07 \\
Noise & 7.55 & 6.06 \\
Other components & 5.53 & 0.31 \\
CMB & 1.45 & 0 \\
Dust + CIB & 5.02 & 0.35 \\
Sync + Free-Free & 0.50 & 0.08 \\
SZ & 0.025 & 0.0020 \\
\hline Maps & CO ILC $\left(30^{\prime}\right)$ & CO MILCA $\left(30^{\prime}\right)$ \\
\hline Total & 5.94 & 9.35 \\
CO & 9.11 & 9.11 \\
Residuals & 4.64 & 0.53 \\
Noise & 0.57 & 0.46 \\
Other components & 4.60 & 0.25 \\
CMB & 1.14 & 0 \\
Dust + CIB & 4.16 & 0.29 \\
Sync + Free-Free & 0.46 & 0.07 \\
SZ & 0.017 & 0.0013 \\
\hline
\end{tabular}

ways in order to reduce significantly residuals from instrumental noise and other astrophysical components. For this purpose the separation is performed both in real and Fourier spaces. Moreover the data covariance matrix is modified and divided into multiple subspaces in order to avoid the confusion between instrumental noise and astrophysical components in the original data. Finally, we have also introduced the possibility of using external templates to improve the efficiency of the algorithm. Thanks to these improvements MILCA has been proved to be more efficient than standard ILC algorithms in a wide range of
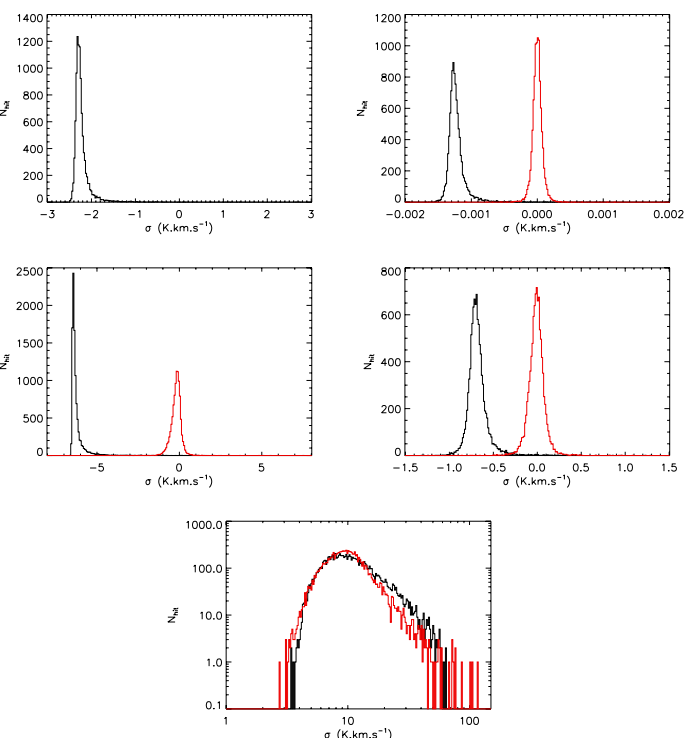

Fig. 10. From left to right and top to bottom: contamination by other components in CO reconstruction for 10000 simulations for CMB, tSZ, Dust + CIB, Synchrotron + Free-Free + Radio point sources, and Noise respectively. In black for a standard CO-adapted ILC and in red for MILCA. Each contamination are presented in terms of standard deviation in units of $\mathrm{K} \mathrm{km} \mathrm{s}^{-1}$.

astrophysical problems. We have also demonstrate the efficiency of our modifications on the ILC estimator to reduce the instrumental noise and the "unwanted" astrophysical components.

We have applied MILCA to simulated Planck data, and have shown that it can be used to efficiently reconstruct the tSZ and CO emissions. We have proposed, with MILCA, an original way to used prior on the spatial distribution of contaminating 
components. Allowing to constraint emissions such as CIB, which can not be described with a single template and an SED.

In the tSZ case we have shown that MILCA can be used to reconstruct low signal-to-noise and highly non-Gaussian components in the Planck data. Indeed, MILCA has been used extensively by the Planck collaboration for tSZ and CO studies. For $\mathrm{CO}$ emission, we have presented a new component separation approach that takes advantage of the spectral bandpass mismatch between bolometers of the same Planck channel.

Acknowledgements. We thank Nabila Aghanim and François-Xavier Désert for useful discussions related to this work. We acknowledge the referee for useful comments that have improved the quality of the paper. Some of the results in this paper have been derived using the HEALPix package (Górski et al. 2005).

\section{References}

Addison, G. E., Dunkley, J., \& Spergel, D. N. 2012, MNRAS, 427, 1741 Arnaud, M., Pratt, G. W., Piffaretti, R., et al. 2010, A\&A, 517, A92 Aumont, J., \& Macías-Pérez, J.-F. 2007, MNRAS, 376, 739 Basak, S., \& Delabrouille, J. 2012, MNRAS, 419, 1163

Bennett, C. L., Banday, A. J., Gorski, K. M., et al. 1996, ApJ, 464, L1 Bennett, C. L., Halpern, M., Hinshaw, G., et al. 2003, ApJS, 148, 1 Bersanelli, M., Mandolesi, N., Butler, R. C., et al. 2010, A\&A, 520, A4 Bobin, J., Moudden, Y., Starck, J.-L., Fadili, J., \& Aghanim, N. 2008, Stat. Methodol., 5, 307

Bobin, J., Starck, J.-L., Sureau, F., \& Basak, S. 2013, A\&A, 550, A73

Bonaldi, A., Bedini, L., Salerno, A., Baccigalupi, C., \& de Zotti. 2006, MNRAS, 373,271

Brunzendorf, J., \& Meusinger, H. 1999, A\&AS, 139, 141

Cardoso, J.-F., Le Jeune, M., Delabrouille, J., Betoule, M., \& Patanchon, G. 2008, IEEE J. Select. Top. Signal Proc., 2, 735

Condon, J. J., Cotton, W. D., Greisen, E. W., et al. 1998, AJ, 115, 1693 Dame, T. M., Hartmann, D., \& Thaddeus, P. 2001, ApJ, 547, 792 Davies, R. D., Watson, R. A., \& Gutierrez, C. M. 1996, MNRAS, 278, 925 Delabrouille, J., Cardoso, J.-F., \& Patanchon, G. 2003, MNRAS, 346, 1089 Delabrouille, J., Betoule, M., Melin, J.-B., et al. 2013, A\&A, 553, A96 Desert, F.-X., Boulanger, F., \& Puget, J. L. 1990, A\&A, 237, 215 Dick, J., Remazeilles, M., \& Delabrouille, J. 2010, MNRAS, 401, 1602 Dickinson, C., Davies, R. D., \& Davis, R. J. 2003, MNRAS, 341, 369 Eriksen, H. K., Banday, A. J., Górski, K. M., \& Lilje, P. B. 2004, ApJ, 612, 633
Eriksen, H. K., Jewell, J. B., Dickinson, C., et al. 2008, ApJ, 676, 10 Finkbeiner, D. P. 2003, ApJS, 146, 407

Górski, K. M., Hivon, E., Banday, A. J., et al. 2005, ApJ, 622, 759

Haslam, C. G. T., Klein, U., Salter, C. J., et al. 1981, A\&A, 100, 209

Hinshaw, G., Spergel, D. N., Verde, L., et al. 2003, ApJS, 148, 135

Hinshaw, G., Weiland, J. L., Hill, R. S., et al. 2009, ApJS, 180, 225

Hobson, M. P., Jones, A. W., Lasenby, A. N., \& Bouchet, F. R. 1998, MNRAS, 300, 1

Lacasa, F., Aghanim, N., Kunz, M., \& Frommert, M. 2012, MNRAS, 421, 1982

Lamarre, J., Puget, J., Ade, P. A. R., et al. 2010, A\&A, 520, A9

Maino, D., Farusi, A., Baccigalupi, C., et al. 2002, MNRAS, 334, 53

Mennella, A., Butler, R. C., Curto, A., et al. 2011, A\&A, 536, A3

Miville-Deschênes, M.-A., \& Lagache, G. 2005, ApJS, 157, 302

Piffaretti, R., Arnaud, M., Pratt, G. W., Pointecouteau, E., \& Melin, J.-B. 2011, A\&A, 534, A109

Planck Collaboration 2006 [arXiv: astro-ph/0604069]

Planck Collaboration 2011a, A\&A, 536, A1

Planck Collaboration 2011b, A\&A, 536, A4

Planck Collaboration 2011c, A\&A, 536, A7

Planck Collaboration 2011d, A\&A, 536, A8

Planck Collaboration 2011e, A\&A, 536, A13

Planck Collaboration 2011f, A\&A, 536, A18

Planck Collaboration 2011g, A\&A, 536, A20

Planck Collaboration 2011h, A\&A, 536, A21

Planck Collaboration 2011i, A\&A, 536, A26

Planck Collaboration 2013a, A\&A, 550, A128

Planck Collaboration 2013b, A\&A, 550, A131

Planck Collaboration 2013c, A\&A, 550, A132

Planck Collaboration 2013d, A\&A, 550, A133

Planck Collaboration 2013e, A\&A, 550, A134

Planck Collaboration 2013f, A\&A, 554, A140

Planck Collaboration 2013g, A\&A, submitted [arXiv: 1303.5062]

Planck Collaboration 2013h, A\&A, submitted [arXiv: 1303.5073]

Planck Collaboration 2013i, A\&A, submitted [arXiv: 1303.5074]

Planck Collaboration 2013j, A\&A, submitted [arXiv: 1303.5075]

Planck Collaboration 2013k, A\&A, submitted [arXiv: 1303.5076]

Planck Collaboration 20131, A\&A, submitted [arXiv: 1303.5081]

Planck Collaboration 2013m, A\&A, submitted [arXiv: 1303.5089]

Remazeilles, M., Delabrouille, J., \& Cardoso, J.-F. 2011, MNRAS, 410, 2481

Smoot, G. F., Bennett, C. L., Kogut, A., et al. 1992, ApJ, 396, L1

Stolyarov, V., Hobson, M. P., Ashdown, M. A. J., \& Lasenby, A. N. 2002, MNRAS, 336, 97

Sunyaev, R. A., \& Zeldovich, Y. B. 1972, Comments Astrophys. Space Phys., 4, 173

Vio, R., \& Andreani, P. 2008, A\&A, 487, 775 